\title{
Early diagnosis of gestational diabetes mellitus using circulating microRNAs
}

\author{
Liron Yoffe', Avital Polsky', Avital Gilam', Chen Raff', Federico Mecacci², Agostino Ognibene ${ }^{3}$, Fatima Crispi ${ }^{4,5}$, \\ Eduard Gratacós ${ }^{4,5}$, Hannah Kanety ${ }^{6}$, Shali Mazaki-Tovi ${ }^{1,7}$, Noam Shomron ${ }^{1}$ and Moshe Hod ${ }^{1,8}$
}

${ }^{1}$ Sackler Faculty of Medicine, Tel Aviv University, Tel Aviv, Israel, ${ }^{2}$ Department of Health Sciences, University of Florence, Obstetrics and Gynecology, Careggi University Hospital, Florence, Italy, ${ }^{3}$ Laboratory Department, Careggi University Hospital, Florence, Italy, ${ }^{4}$ Fetal Medicine Research Center, BCNatal - Barcelona Center for Maternal-Fetal and Neonatal Medicine (Hospital Clínic and Hospital Sant Joan de Deu), Institut Clínic de Ginecologia, Obstetricia i Neonatologia, Institut d'Investigacions Biomèdiques August Pi i Sunyer, Universitat de Barcelona, Barcelona, Spain, ${ }^{5}$ Center for Biomedical Research on Rare Diseases (CIBERER), Barcelona, Spain, ${ }^{6}$ Institute of Endocrinology, Sheba Medical Center, Tel-Hashomer, Ramat-Gan, Israel, ${ }^{7}$ Department of Obstetrics and Gynecology, Sheba Medical Center, Tel-Hashomer, Ramat-Gan, Israel, and ${ }^{8}$ Rabin Medical Center,

Correspondence should be addressed to N Shomron

Email

nshomron@post.tau.ac.il

\section{Abstract}

Design: Gestational diabetes mellitus (GDM) is one of the most common pregnancy complications and its prevalence is constantly rising worldwide. Diagnosis is commonly in the late second or early third trimester of pregnancy, though the development of GDM starts early; hence, first-trimester diagnosis is feasible.

Objective: Our objective was to identify microRNAs that best distinguish GDM samples from those of healthy pregnant women and to evaluate the predictive value of microRNAs for GDM detection in the first trimester.

Methods: We investigated the abundance of circulating microRNAs in the plasma of pregnant women in their first trimester. Two populations were included in the study to enable population-specific as well as cross-population inspection of expression profiles. Each microRNA was tested for differential expression in GDM vs control samples, and their efficiency for GDM detection was evaluated using machine-learning models.

Results: Two upregulated microRNAs (miR-223 and miR-23a) were identified in GDM vs the control set, and validated on a new cohort of women. Using both microRNAs in a logistic-regression model, we achieved an AUC value of 0.91. We further demonstrated the overall predictive value of microRNAs using several types of multivariable machine-learning models that included the entire set of expressed microRNAs. All models achieved high accuracy when applied on the dataset (mean AUC $=0.77$ ). The significance of the classification results was established via permutation tests. Conclusions: Our findings suggest that circulating microRNAs are potential biomarkers for GDM in the first trimester. This warrants further examination and lays the foundation for producing a novel early non-invasive diagnostic tool for GDM.

\section{Introduction}

Gestational diabetes mellitus (GDM) is a pregnancy complication that is formally defined as 'condition in which carbohydrate intolerance develops during pregnancy' (1). The carbohydrate intolerance can be of variable severity (2). GDM can affect $1-20 \%$ of pregnancies depending on the diagnostic criteria used (c) 2019 European Society of Endocrinology Printed in Great Britain
European Journal of Endocrinology

(2019) 181, 565-577 and on the population (3). In normal pregnancy, during the first trimester insulin resistance may be unchanged, increased, or even decreased $(4,5,6)$. However, during the second and third trimesters insulin resistance increases by $40-60 \%$ in order to ensure appropriate glucose supply for the fetus $(4,5,7,8,9,10)$. This is accompanied by 
compensatory augmentation in insulin production and/or secretion to maintain glucose homeostasis (11). In GDM, glucose intolerance develops most likely due to reduced insulin sensitivity, without the ability to compensate by increasing insulin secretion (12).

Multiple clinical risk factors for GDM have been established including obesity, increased maternal age, family history of type 2 diabetes, past history of GDM, a previous adverse pregnancy outcome and belonging to a high-risk ethnic group (13). Nevertheless, risk factors alone are insufficient to accurately predict risk for GDM development (14).

GDM is associated with considerable short and long term risks for both the mother and the offspring (1). Maternal risks include a greater likelihood of undergoing a caesarian section, the development of preeclampsia, and the post-pregnancy development of type 2 diabetes and cardiovascular disease in adulthood $(15,16)$. Fetal risks include macrosomia or large-for-gestational-age neonates, shoulder dystocia at delivery, neonatal hypoglycemia, and obesity with insulin resistance in young adulthood $(17,18$, 19). These potential adverse outcomes in both mother and offspring reinforce the importance of correctly diagnosing and managing GDM. GDM management traditionally includes dietary modification and pharmacological therapies such as metformin and insulin, both have been shown to reduce immediate adverse outcomes $(20,21)$.

In most developed countries, GDM is screened and diagnosed in the late second or early third trimester of pregnancy (24-28 weeks of gestation). The reason for performing the test in late stages of the pregnancy is that diabetogenic effects increase with gestation, and therefore, testing at later stages of pregnancy should maximize the detection rate (22). However, according to the recent guidance of the American Congress of Obstetricians and Gynecologists (ACOG), early screening in the first trimester is recommended in women with risk factors (e.g., BMI above 25, hypertension, known impaired glucose metabolism, and family history of diabetes) (23). Two diagnostic strategies are the $75 \mathrm{~g}$ oral glucose tolerance test (OGTT) and the $50 \mathrm{~g}$ screening test followed by a $100 \mathrm{~g}$ OGTT for those with a positive test (24). These tests are time-consuming, labor-intensive, and poorly tolerated by pregnant women. Physiological discomfort leads to approximately $10 \%$ of women not completing the tests, with vomiting during the test being the major reason (25).

Current studies show that GDM management after diagnosis at 24-28 weeks can be beneficial in controlling short-term complications (e.g. macrosomia, large for gestational age, shoulder dystocia, and pre-eclampsia/ hypertensive disorders in pregnancy), but not long-term complications $(20,21,26)$. Effective early identification, in the first trimester, of the development of GDM might reduce disease onset and associated maternal and perinatal complications. Additionally, an effective early diagnosis test will enable to carry large scale prospective studies for the evaluation of early GDM treatment. Applying GDM screening (i.e., 50g) and diagnosis (i.e., OGTT) tests in the first trimester has been shown to be useful when the currently used second-trimester cutoffs are lowered for both tests (27). Nevertheless, since the incidence of nausea and vomiting is at its highest in the first trimester, such tests may increase maternal discomfort and may result in an even higher failure rate. Thus, an early noninvasive, easily tolerated screening and diagnostic tool for GDM, may reduce maternal discomfort, and thus, yield an increased success rate.

The identification of early pregnancy biomarkers, readily obtained from blood samples, may complement existing clinical risk factors in detecting women at high risk of developing GDM (14). Over the years, numerous attempts have been made to identify biomarkers for GDM early in pregnancy such as adiponectin (an adipocytederived hormone) (28), glycosylated hemoglobin (HbA1c) $(29,30,31,32)$ as well as several additional factors including metabolic biomarkers (e.g., sex hormone-binding globulin (33)), inflammatory biomarkers (e.g., tumor necrosis factor alpha (34)), and placental biomarkers (e.g. glucose transporters, GLUTs (35)); currently, none have sufficient validity for clinical practice.

MicroRNAs (miRNAs) are untranslated small RNA molecules that regulate gene expression by facilitating mRNA degradation or by inhibiting protein translation (36). The role of miRNAs in human disease has been investigated extensively $(37,38,39,40,41)$, and their potential to act as biomarkers was well studied in several conditions, particularly in cancer (42). In the context of diabetes, circulating miRNAs were investigated as potential early biomarkers mainly for the diagnosis of type 1 diabetes and type 2 diabetes (43), and a few potential miRNA biomarkers were suggested $(44,45)$. Moreover, miRNAs have been associated with several mechanisms related to diabetes pathogenesis including $\beta$ cell function and regulation, and immune system homeostasis $(46,47,48)$. In recent years, the expression of circulating miRNAs in GDM was investigated, as well as their potential to serve as GDM diagnosis biomarkers. Nevertheless, the studies that have been conducted thus far have focused on later stages of the pregnancy 
(early second trimester until full term pregnancy), rather than on the first trimester. Zhao et al. reported downregulations of miR-132, miR-29a, and miR-222 in serum collected between 16 and 19 gestational weeks from women who developed GDM vs normoglycemic controls (49). A few other studies explored miRNA expression in maternal plasma/serum (50), in placenta samples (51, 52 ), in omental adipose tissue biopsies (collected at the time of caesarian section) (53), and in cultured human umbilical vein endothelial cells $(54,55)$. Nevertheless, no miRNA marker has demonstrated a consistent association or has been implemented in clinical practice. Moreover, understanding miRNA function could improve the understanding of the etiology and pathophysiology of GDM.

In the current study, we investigated the abundance of circulating miRNAs in the plasma of pregnant women in their first trimester, while seeking miRNAs that best distinguish GDM and control groups and that can thus serve as potential early diagnostic biomarkers for GDM. To increase the generalizability of our analysis, samples were collected from women residing in two countries: Italy and Spain.

\section{Subjects and methods}

\section{Collection of plasma samples}

This is a case-control study of women who were attending routine visits during pregnancy. At a visit that was held at $9^{+0}$ to $11^{+6}$ weeks gestation, maternal characteristics, medical history and clinical measurements were recorded and blood samples were collected. The study consisted of 43 pregnant women: 23 women who developed GDM and 20 healthy women with uncomplicated pregnancies (i.e., a control set). Blood samples from Italy were collected at Careggi University Hospital in 2014 after overnight fasting or after a light breakfast. The samples were centrifuged, frozen at $-20^{\circ} \mathrm{C}$ for a month, and subsequently stored at $-80^{\circ} \mathrm{C}$. Blood samples from Spain were collected from October 2006 to January 2012 and were obtained from the biobank of the August Pi i Sunyer Biomedical Research Institute. The samples were collected after at least $4 \mathrm{~h}$ of fasting and were stored at $-80^{\circ} \mathrm{C}$. The samples from women with GDM were selected at random, and each was matched to a control sample that was extracted on the same or next day. All women of the control set delivered at term phenotypically normal neonates and with birth weights between the 5th and 95th percentiles for gestational age at delivery. None of these women had a history of diabetes. Women at the GDM group who had pre-gestational diabetes or other diseases affecting carbohydrate metabolism were excluded. The women gave written informed consent for the use of their blood samples in research, and the study was approved by the ethics committee of the University Hospital Clinic de Barcelona, Spain; and by the Ethics Committee of the University Hospital of Siena, Italy. All methods were performed in accordance with the relevant guidelines and regulations. Data on pregnancy outcomes were abstracted from the hospital maternity records or the general medical practitioners of the women.

All participants in the Italian cohort underwent a $75 \mathrm{~g}$ OGTT at 22-24 weeks gestation. Venous blood samples were obtained at 0,1 , and $2 \mathrm{~h}$ for determination of plasma glucose. GDM was diagnosed according to the International Association of Diabetes and Pregnancy Study Groups (IADPSG) diagnostic criteria for GDM (24): fasting glucose $\geq 5.11 \mathrm{mmol} / \mathrm{L}, \quad 1$-h glucose $\geq 10.00 \mathrm{mmol} / \mathrm{L}$, and 2-h glucose $\geq 8.50 \mathrm{mmol} / \mathrm{L}$. GDM was diagnosed if at least one value was elevated. Women from the Spanish cohort underwent an O'Sullivan screening test $(50 \mathrm{~g})$ at 24 -week gestation. Then, if abnormal (i.e., glucose $>7.8 \mathrm{mmol} / \mathrm{L}$ ), a $100 \mathrm{~g}$ OGTT was performed at 26-week gestation. GDM was diagnosed if two values were elevated: fasting glucose $\geq 5.30 \mathrm{mmol} / \mathrm{L}, \quad 1-\mathrm{h}$ glucose $\geq 10.00 \mathrm{mmol} / \mathrm{L}, \quad 2-\mathrm{h}$ glucose $\geq 8.60 \mathrm{mmol} / \mathrm{L}$, and 3 -h glucose $\geq 7.80 \mathrm{mmol} / \mathrm{L}$.

\section{Collection of placenta and adipose samples}

Paired specimens of placenta, visceral adipose tissue, and abdominal subcutaneous adipose tissue were collected at Sheba Medical Center, Israel. Samples were obtained post-delivery from six women who developed GDM and from eight healthy pregnant women with uncomplicated pregnancies. GDM was diagnosed according to the O'Sullivan screening test $(50 \mathrm{~g})$ at 24 -week gestation, following a 100-g OGTT at 26-week gestation.

\section{RNA extraction and miRNA profiling}

RNA was extracted via miRNeasy Serum/Plasma Kit. RNA yields were measured using the Qubit 2.0 Fluorometer (Life Technologies) in combination with the microRNA Assay Kit (Life Technologies) according to the manufacturer's protocol. The multiplexed NanoString nCounter miRNA expression assay (NanoString Technologies, Seattle, 
USA) (56) was used to profile 798 human miRNAs. The assay was performed according to the manufacturer's protocol, with the exception of the RNA input, whichdue to low RNA concentrations-was $4 \mu \mathrm{L}$. Briefly, $4 \mu \mathrm{L}$ of total RNA was used as input material, with $3 \mu \mathrm{L}$ of the threefold-diluted sample. A specific DNA tag was ligated to the 3 ' end of each mature miRNA, providing exclusive identification for each miRNA species in the sample. The tagging was performed in a multiplexed ligation reaction utilizing reverse complementary bridge oligonucleotides to achieve ligation of each miRNA to its designated tag. All hybridization reactions were incubated at $64^{\circ} \mathrm{C}$ for $18 \mathrm{~h}$. Excess tags were then removed, and the resulting material was hybridized with a panel of fluorescently labeled, bar-coded reporter probes specific to the miRNA of interest. Abundances of miRNAs were quantified on the nCounter Prep Station by counting individual fluorescent barcodes and quantifying target miRNA molecules present in each sample. The highest expression value of the negative controls was set as the lower threshold for the miRNAs and was subtracted from their expression values. A miRNA with 1 or less total counts after the negative control subtraction was excluded.

\section{Differential expression analysis}

We first performed Principal Component Analysis (PCA) using prcomp method in R. A PCA plot was used to discover unwanted variation present in the data (i.e., batch effects) and to detect outlier samples. This analysis indicated the existence of a batch effect that matched the dates of the processing and sequencing of the samples. We applied DESeq2 (57) (in R) to obtain a list of differentially expressed miRNAs between control and GDM samples. The differential expression test was done while controlling for obesity, the source country of the samples and the technical batch covariates. Only miRNAs with $P$ value $<0.05$ after multiple test corrections were considered.

\section{Sample classification}

Machine learning models were trained and evaluated on three datasets via a leave-one-out cross-validation (LOOCV) procedure. In each cycle of the LOOCV, a model was trained on $\mathrm{n}-1$ samples (where $\mathrm{n}$ is the number of samples) and tested on the remaining sample. This process was repeated $\mathrm{n}$ times; thus, all samples were used as a test set. Three types of classification models were used: logistic regression, random forest
(58), and AdaBoost (59). The model features included either the differentially expressed miRNAs or the entire set of miRNAs with detectible expression in the samples (i.e. total counts $\geq 1$ ). We applied a two-step feature selection procedure in each of the LOOCV cycles: first collapsing highly correlated miRNAs (i.e., Pearson correlation $>0.75$ ), and then removing miRNAs with poor correlation to the sample group (i.e., GDM/control, Pearson correlation $<0.4)$. Logistic regression models were further limited to up to five miRNAs, to avoid over fitting. AdaBoost and random forest models were limited to a minimum of ten miRNAs. Permutation tests were performed using the same procedure with additional a priori permutations of the samples' group labels (i.e. GDM/control). Each permutation test comprised 100 iterations of randomly permuting sample group labels, together with the training of a model on the permuted dataset. We then compared the results obtained from the original dataset to the distribution of the results obtained from the permutation test. $P$ values for the performance of the model on the original dataset were evaluated using the Monte-Carlo method (60): $(r+1) /(m+1)$, where $m$ is the number of permutation test iterations and $r$ is the number of these iterations that produced a test statistic greater than or equal to that calculated for the original data. Counts were normalized using DESeq2 (57) package in $\mathrm{R}$, and batch effects were removed using the removeBatchEffect method from limma package (61), which adjusts for known batches using a linear model. The classification pipeline was implemented in python using the scikit-learn library (62).

\section{Quantitative polymerase chain reaction (qPCR) validations}

RT-qPCR was performed to validate the differentially expressed miRNAs obtained by NanoString nCounter in GDM and control samples. RNA was extracted via miRNeasy Serum/Plasma Kit. The Applied Biosystems ${ }^{\mathrm{TM}}$ TaqMan ${ }^{\mathrm{TM}}$ Advanced miRNA Assay (Applied Biosystems; Thermo Fisher Scientific, Inc.) was used to test miRNA expression. PCR amplification and reading were performed with the StepOne ${ }^{\mathrm{TM}}$ Real-Time PCR System (Life Technologies; Thermo Fisher Scientific, Inc.). Mature miRNA expression was quantified under the following thermal cycler conditions: $2 \mathrm{~min}$ at $50^{\circ} \mathrm{C}$, $10 \mathrm{~min}$ at $95^{\circ} \mathrm{C}$ and 40 amplification cycles $\left(15 \mathrm{~s}\right.$ at $95^{\circ} \mathrm{C}$ and $1 \mathrm{~min}$ at $60^{\circ} \mathrm{C}$ ). Expression values were calculated based on the comparative threshold cycle method (63), and normalized with cel-miR-39 spike-in. One outlier 
sample was removed from the analysis due to anomalous results in RT-qPCR.

\section{Results}

\section{Patient characteristics}

RNA was extracted and miRNAs were profiled from the plasma of 43 pregnant women: 23 who developed GDM and 20 normoglycemic women with uncomplicated pregnancies (i.e., a control set). All women were in their first trimester (gestational age $9^{+0}-11^{+6}$ weeks). To account for the contribution of obesity to the risk of GDM, 11 samples were obtained from obese women (BMI $>30 \mathrm{~kg} /$ $\mathrm{m}^{2}$ ): six of them developed GDM and five had healthy pregnancies. Samples were collected in clinics in two countries: Italy and Spain. All the women in the control group were without pre-existing medical conditions, with uncomplicated pregnancies that resulted in a delivery of a phenotypically normal neonate at term and with normal birthweight for gestational age at delivery. Cases and controls were matched regarding country of residence, pre-pregnancy maternal body mass index (BMI), maternal obesity, fetal gender, gestational age at delivery, and birth weight. They differed significantly regarding fasting glucose $(P$ value $<0.0001)$, 1-h glucose $(P$ value $<0.0001)$, and 2 -h glucose $(P$ value $=0.0019)$, which are all values of the OGTT that were used in GDM diagnosis (see a summary of maternal and pregnancy characteristics in Table 1).

\section{Characterizing miRNA expression profiles in GDM and control groups}

The miRNA expression profiles in GDM and control samples were characterized using NanoString nCounter human miRNA assay (56). Due to extremely low numbers of expressed miRNAs or to large anomalies in expression profiles, 22 samples were removed from downstream analysis. Each of the miRNAs was tested for differential expression in GDM vs control samples and two miRNAs: miR-223 and miR-23a were found to be significantly upregulated in the GDM group (adjusted $P$ value $=1.4 \times 10^{-7}$ and 0.019 respectively, Table 2 and Fig. 1). To further examine miRNA expression in the GDM vs control group at a higher resolution, we analyzed the data by country of residence and examined the miRNAs for differential expression in the limited datasets. In the Spain dataset, miR-223 was significantly upregulated in GDM vs control samples (adjusted $P$ value $=0.014$ ), and in the Italy dataset, both miR-223 and miR-23a were upregulated in GDM vs control samples (adjusted $P$ values $=0.0003$ and 0.006, respectively).

\section{Validations for the differentially expressed miRNAs}

To validate the differentially expressed miRNAs, we examined expression of miR-223 and miR-23a in a new cohort using RT-qPCR. The new cohort comprised samples obtained from 20 women from Italy: ten GDM patients

Table 1 Maternal and pregnancy characteristics.

\begin{tabular}{l}
\hline Characteristics \\
\hline Maternal age, years ${ }^{*}$ \\
Gestational age at sampling, weeks* \\
Gestational age at delivery, weeks \\
Body mass index, $\mathrm{kg} / \mathrm{m}^{2 *}$ \\
Fasting glucose, $\mathrm{mmol} / \mathrm{L}^{*}$ \\
1-h glucose, $\mathrm{mmol} / \mathrm{L}^{*}$ \\
2-h glucose, $\mathrm{mmol} / \mathrm{L}^{*}$ \\
Birth weight, $\mathrm{g}^{*}$ \\
Fetal gender, $n(\%)$ \\
Female \\
Male \\
Country of residence, $n(\%)$ \\
Italy \\
Spain \\
Obesity, $n(\%)$ \\
Yes \\
No
\end{tabular}

*Data are reported as medians (interquartile ranges).

$P$ values were calculated using Fisher's exact test for categorical variables, and using the Mann-Whitney $U$ test for continuous variables. Statistically significant differences are marked in bold.

\begin{tabular}{c}
\hline Control $(n=20)$ \\
\hline $34.5(32.0-37.2)$ \\
$10.0(10.0-10.2)$ \\
$39.9(39.4-40.7)$ \\
$22.2(20.1-31.0)$ \\
$4.1(4.1-4.6)$ \\
$7.0(6.1-8.1)$ \\
$6.1(5.2-6.5)$ \\
$3260(3106-3410)$ \\
$9(45.0)$ \\
$11(55.0)$ \\
$11(55.0)$ \\
$9(45.0)$ \\
$5(45.5)$ \\
$6(54.5)$
\end{tabular}

\begin{tabular}{crrr}
\hline GDM $(n=23)$ & & P value \\
\hline $34.0(32.5-37.5)$ & & 0.9124 \\
$10(10.0-10.6)$ & & 0.2264 \\
$40.3(38.4-40.7)$ & & 0.6803 \\
$28.6(20.4-31.1)$ & & 0.9840 \\
$5.2(4.9-5.3)$ & & $<\mathbf{0 . 0 0 0 1}$ \\
$9.0(8.2-10.4)$ & & $<\mathbf{0 . 0 0 0 1}$ \\
$7.3(6.1-8.5)$ & & $\mathbf{0 . 0 0 1 9}$ \\
$3555(3060-4016)$ & & 0.1659 \\
& & 1.0000 \\
$11(47.8)$ & \\
$12(52.2)$ & \\
$13(56.5)$ & \\
$10(43.5)$ & \\
$7(50.0)$ & \\
$7(50.0)$ & \\
\hline
\end{tabular}


Table 2 Differentially expressed miRNAs in GDM vs control groups.

\begin{tabular}{|c|c|c|}
\hline Dataset & Country & MiRNA \\
\hline 1 & Spain and Italy & $\begin{array}{l}\text { miR-223-3p } \\
\text { miR-23a-3p }\end{array}$ \\
\hline 2 & Italy & $\begin{array}{l}\text { miR-223-3p } \\
\text { miR-23a-3p }\end{array}$ \\
\hline 3 & Spain & miR-223-3p \\
\hline
\end{tabular}

and ten healthy women with uncomplicated pregnancies (Table 3). Both miRNAs were significantly upregulated in this dataset as well in GDM vs control samples (miR-223, $P$ value $=0.009 ;$ miR-23a, $P$ value $=0.03)$, which validated the differential expression observed in the original datasets (Fig. 2).

\section{miRNA-based classification of samples}

To evaluate the predictive value of the differentially expressed miRNAs we utilized a machine-learning model (i.e., logistic regression), which classifies GDM and control samples based on the expression values of the differentially expressed miRNAs. Three models were utilized: one model included both miR-223 and miR-23a, and the other two models included one of the two miRNAs each. Comparing the models by the AUC values, miR-223 was a slightly better classifier than miR-223 + miR-23a or miR-23a alone: $\mathrm{AUC}=0.94$ and accuracy $=0.90$ for miR-223; $\mathrm{AUC}=0.89$ and accuracy $=0.90$ for miR-23a; and $\mathrm{AUC}=0.91$ and accuracy $=0.90$ for $\mathrm{miR}-223+$ miR-23a (Table 4 ). To evaluate the significance of the classification results, we performed permutation tests that compare the accuracy of the model to the accuracy of a random classifier (see details in Materials and methods). The AUC and accuracy values for all trained models were significant $(P$ value $<0.05)$, as were most other statistical measures for performance evaluation (e.g., F1 score, Matthews's correlation coefficient, and positive likelihood ratio, Table 4).

Due to its complex nature, GDM is most likely related to differences in expression of several more miRNAs, for which statistical significance of the differential expression was not attained due to underpowered statistical tests. Hence, we expanded our search to miRNA-based classification models that do not rely entirely on differentially expressed miRNAs, but rather included less-discriminating miRNAs as well. Considering a wider range of models enabled us to assess the general predictive value of circulating miRNAs in the first trimester. Thus, we utilized a multivariable model that combines the ability of several miRNAs to differentiate the samples in a synergic manner. To this

\begin{tabular}{|c|c|c|}
\hline Mean counts & log2 fold change & Adj. P value \\
\hline 185.22 & 5.46 & $1.42 \times 10^{-7}$ \\
\hline 111.98 & 3.04 & $1.92 \times 10^{-2}$ \\
\hline 65.52 & 5.61 & $2.56 \times 10^{-4}$ \\
\hline 39.76 & 4.40 & $6.61 \times 10^{-3}$ \\
\hline 463.90 & 4.88 & $1.42 \times 10^{-2}$ \\
\hline
\end{tabular}

end, we trained three classification model types (i.e., logistic regression, random forest, and AdaBoost). Applying the classification models on the dataset, all models achieved accuracy $>0.81$ and AUC $>0.74$. We performed a separate permutation test for each of the

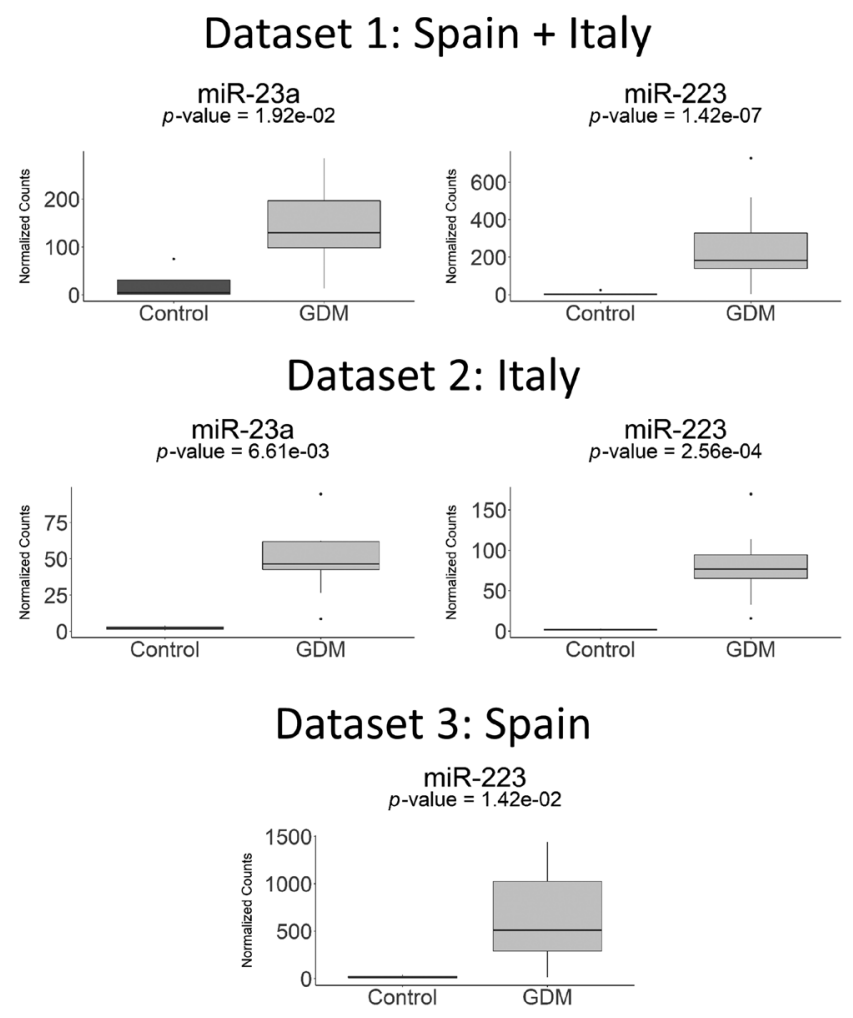

Figure 1

Differentially expressed miRNAs in GDM vs. control samples. Each of the expressed miRNAs in GDM vs control samples was tested for differential expression in the entire dataset, as well as in both subsets by the county in which the samples were collected: Spain and Italy. Two miRNAs were found to be differentially expressed (adjusted $P$ value $<0.05$ ). Normalized counts of the differentially expressed miRNA are presented as box plots. The upper and lower limits of the boxes represent the $75^{\text {th }}$ and $25^{\text {th }}$ percentiles. The upper and lower whiskers represent maximum and minimum values. The median is indicated by the line in each box. Outliers are indicated by circles. 
Table 3 Maternal and pregnancy characteristics of the validation cohort.

\begin{tabular}{l} 
Characteristics \\
\hline Maternal age, years* \\
Gestational age at sampling, weeks* \\
Gestational age at delivery, weeks \\
Body mass index, $\mathrm{kg} / \mathrm{m}^{2 *}$ \\
Fasting glucose, $\mathrm{mmol} / \mathrm{L}^{*}$ \\
1-h glucose, $\mathrm{mmol} / \mathrm{L}^{*}$ \\
2-h glucose, $\mathrm{mmol} / \mathrm{L}^{*}$ \\
Birth weight, g* \\
Fetal gender, $n(\%)$ \\
Female \\
Male \\
Obesity, $n(\%)$ \\
Yes \\
No
\end{tabular}

\begin{tabular}{c}
\hline Control $(n=10)$ \\
\hline $35.5(33.0-37.8)$ \\
$10.0(10.0-10.0)$ \\
$39.7(39.1-40.4)$ \\
$22.5(20.5-30.9)$ \\
$4.4(4.3-4.4)$ \\
$7.2(6.9-8.5)$ \\
$6.0(5.5-6.6)$ \\
$3175(3010-3267)$ \\
$3(30.0)$ \\
$7(70.0)$ \\
$4(40.0)$ \\
$6(60.0)$
\end{tabular}

\begin{tabular}{c}
\hline $\mathbf{G D M}(n=10)$ \\
\hline $36.5(32.2-39.8)$ \\
$10(10.0-10.0)$ \\
$40.6(39.5-41.0)$ \\
$27.8(20.9-33.1)$ \\
$5.2(4.7-5.3)$ \\
$11.1(10.1-12.0)$ \\
$9.3(8.0-9.6)$ \\
$3485(3272-3585)$ \\
$5(50.0)$ \\
$5(50.0)$ \\
$5(50.0)$ \\
$5(50.0)$
\end{tabular}

\begin{tabular}{l}
\hline P value \\
\hline 1.0000 \\
1.0000 \\
0.4050 \\
0.4055 \\
$\mathbf{0 . 0 0 0 6}$ \\
$\mathbf{0 . 0 0 0 2}$ \\
$\mathbf{0 . 0 0 1 1}$ \\
0.0892 \\
0.6499 \\
\\
\end{tabular}

*Data are reported as medians (interquartile ranges).

$P$ values were calculated using Fisher's exact test for categorical variables, and using the Mann-Whitney $U$ test for continuous variables.

Statistically significant differences are marked in bold.

three model types and obtained significant results for all three models (Table 4). The random-forest and AdaBoost models obtained similar results with slightly better AUC value for the random forest model $(0.81, P$ value $=0.02)$, and slightly better accuracy for the AdaBoost model $(0.86, P$ value $=0.01)$. Figure 3 displays the classification results of the random forest model that was trained on the original dataset vs the classification results of the permutation tests on the same dataset.

\section{The expression of miR-223 and miR-23a in the placenta and in adipose tissues}

The miRNA expression changes identified in the plasma most likely originated from different organs and tissues involved in GDM etiology. We examined the expression of the differentially expressed miRNAs identified in the plasma in three other GDM related tissues. The aim was to assess the source of the differentially expressed miRNAs. The three tissues examined were placental tissue, visceral adipose tissue, and abdominal subcutaneous adipose tissue. Paired specimens were collected post-delivery from six women who developed GDM and from eight healthy women with uncomplicated pregnancies (Table 5). The miRNA expression profile was characterized via NanoString nCounter human miRNA assay (56). Of the 798 human miRNAs in the assay, nearly 400 were expressed in each tissue type. MiR-223 and miR-23a were both highly expressed in all samples, though they were not differentially expressed between the GDM and control groups (Fig. 4).

\section{Discussion}

miRNAs are involved in various pregnancy and GDM related mechanisms. Additionally, circulating miRNAs are stable and detectible in maternal blood; hence, they are promising candidate biomarkers for noninvasive diagnosis tests of pregnancy complications. In this study we assessed the usefulness of circulating miRNAs for firsttrimester prediction of GDM. To this end, we characterized the expression of miRNAs in maternal plasma and examined each miRNA for differential expression between women who subsequently developed GDM and healthy

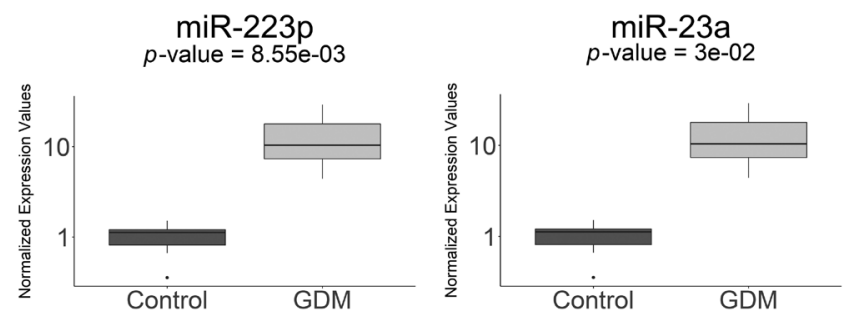

\section{Figure 2}

Validations for the upregulated miRNAs. RT-qPCR was performed on a new cohort of 20 samples: ten GDM samples and ten control samples. RT-qPCR relative expression values $\left(2^{-\Delta C t}\right)$ of miR-223 and miR-23a are presented as box plots. Counts were normalized to obtain an average expression of 1 in the control group. The upper and lower limits of the boxes represent the $75^{\text {th }}$ and $25^{\text {th }}$ percentiles. The upper and lower whiskers represent maximum and minimum values. The median is indicated by the line in each box. Outliers are indicated by circles. 
Table 4 Classification results. Classification results obtained via logistic regression (LR), random forest (RF) and AdaBoost models.

\begin{tabular}{l} 
Model \\
\hline RF \\
AdaBoost \\
LR \\
LR: miR-223 \\
LR: miR-23a \\
LR: miR-223 + mir-23a
\end{tabular}

\begin{tabular}{|c|c|}
\hline AUC & Acc \\
\hline $0.81^{\star \star}$ & $0.81^{* *}$ \\
\hline $0.77^{\star \star \star}$ & $0.86^{* * *}$ \\
\hline $0.74^{\star *}$ & $0.76^{*}$ \\
\hline $0.94^{\star * *}$ & $0.90^{* * *}$ \\
\hline $0.89^{* * *}$ & $0.90^{* * *}$ \\
\hline $0.91^{\text {***}}$ & $0.90^{* * *}$ \\
\hline
\end{tabular}

\begin{tabular}{l}
\hline \multicolumn{1}{c}{ Sens } \\
\hline 0.94 \\
$0.94^{*}$ \\
$0.88^{* \star}$ \\
$0.94^{*}$ \\
$1.00^{\star \star \star}$ \\
$0.94^{*}$ \\
\hline
\end{tabular}

\begin{tabular}{l}
\hline \multicolumn{1}{c}{ Spec } \\
\hline 0.40 \\
$0.60^{\star}$ \\
0.40 \\
$0.80^{* \star *}$ \\
$0.60^{\star}$ \\
$0.80^{* \star *}$ \\
\hline
\end{tabular}

\begin{tabular}{l}
\hline F1 Score \\
\hline $0.88^{\star \star}$ \\
$0.91^{\star \star \star}$ \\
$0.85^{\star \star}$ \\
$0.94^{\star * \star}$ \\
$0.94^{\star \star *}$ \\
$0.94^{\star \star *}$ \\
\hline
\end{tabular}

\begin{tabular}{l}
\hline MCC \\
\hline $0.41^{* \star}$ \\
$0.58^{* \star *}$ \\
0.30 \\
$0.74^{* \star *}$ \\
$0.73^{* * *}$ \\
$0.74^{* \star *}$ \\
\hline
\end{tabular}

\begin{tabular}{l}
\hline PLR \\
\hline $1.56^{* *}$ \\
$2.34^{* * *}$ \\
1.46 \\
$4.69^{* * *}$ \\
$2.50^{* * *}$ \\
$4.69^{* * *}$ \\
\hline
\end{tabular}

\begin{tabular}{l}
\hline \multicolumn{1}{c}{$\mathbf{N L R}$} \\
\hline $0.16^{\star \star}$ \\
$0.10^{\star \star \star}$ \\
$0.31^{*}$ \\
$0.08^{* \star \star}$ \\
$0.00^{\star \star \star}$ \\
$0.08^{\star \star \star}$ \\
\hline
\end{tabular}

$\star P$ value $\leq 0.1 ; * * P$ value $\leq 0.05 ; * * * P$ value $\leq 0.01$

Acc, accuracy, true classifications out of all classifications; AUC, area under the ROC curve; F1 score, the harmonic mean of precision and sensitivity; MCC: Matthews's correlation coefficient, a correlation coefficient between the observed and predicted binary classifications; NLR, negative likelihood ratio (1 - sensitivity) / specificity; PLR, positive likelihood ratio, sensitivity /(1 - specificity); Sens, sensitivity, true positives out of all positives; Spec, specificity, true negatives out of all negatives.

women with uncomplicated pregnancies. The inclusion of pregnant women from two countries, Italy and Spain, increases the generalizability of our results. Two miRNAs were found to be significantly upregulated in the GDM samples: miR-223 and miR-23a. In a subset of the GDM and control samples collected in Italy, both miRNAs remained significantly upregulated, and significant upregulation of miR-223 was also observed in the subset of samples collected in Spain. Both miRNAs were also further validated on a new cohort of 20 women from Italy via RT-qPCR.

MiR-223 affects various cellular processes, ranging from cell cycle regulation and invasiveness to hematopoietic differentiation and immune cell function. As a consequence of its cellular functions, miR-223 expression is deregulated in a wide range of pathologies, such as inflammatory disorders (e.g., rheumatoid arthritis (64) and inflammatory bowel disease (65)), infection (e.g., influenza (66) and hepatitis B infection (67)), and various types of cancer (e.g., leukemia (68) and lymphomas (69)). Previous studies found downregulation of circulating miR-223 in type 2 diabetes. On the other hand, miR223 was found to be upregulated in the insulin resistant heart of type 2 diabetes patients (70). Overexpression of miR-223 was found to enhance glucose uptake to cardiomyocytes by post-transcriptional upregulation of glucose transporter 4 (Glut4); this supports a role of miR223 in Glut4 regulation and in glucose metabolism in the heart (70). In a recently reported meta-analysis that included 39 case-control studies, miR-223 was suggested as a potential circulating biomarker of type 2 diabetes (71). Additionally, an association was described between the expression of circulating miR-223 in the beginning of the second trimester and the subsequent risk of GDM among mothers bearing a male offspring (72). MiR-23a, which was upregulated in GDM samples as well, is known to be involved in the onset of diverse cardiovascular and cerebrovascular diseases (73). Moreover, circulating miR-23a was suggested as a potential biomarker for early diagnosis of pre-diabetes and type 2 diabetes and was found to be particularly useful in discriminating undiagnosed diabetes from pre-diabetes (74).

With the aim of identifying the organ of origin at the basis of the differences in the expression of miR-223 and miR-23a in plasma, we examined their expression in placenta and adipose tissue samples. However, dysregulation of these miRNAs was not identified in the inspected tissues. The dissimilarity in expression between miRNAs in the plasma and those in the placenta and adipose tissue suggests that the altered expression in GDM originates in another tissue. Other possible explanations are different expression profiles in the first vs the third trimester, and population-specific differences. More placenta and adipose samples of GDM patients and healthy pregnant women are required to examine this issue further.

To evaluate the predictive value of the differentially expressed miRNAs we used logistic regression classification models via a LOOCV procedure. We evaluated the significance of the classification results by comparing them to the results obtained by applying the same procedure in 100 iterations of random assignments of the samples into the GDM and control groups. The classification models obtained using this procedure were all highly and significantly accurate. We concluded that utilizing the differentially expressed miRNAs for classification models is highly effective for GDM prediction. Nevertheless, due to its heterogeneous and complex nature, GDM might not be accurately detected by a single or a couple of variables. This could result in decreased accuracy when applying the classification model to larger datasets. A multivariable model that incorporates the capability of several miRNAs to classify GDM is more likely to reach clinically useful efficiency levels. Hence, we further explored GDM 


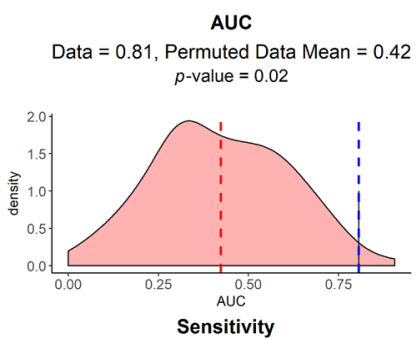

Data $=0.94$, Permuted Data Mean $=0.8$ $p$-value $=0.13$

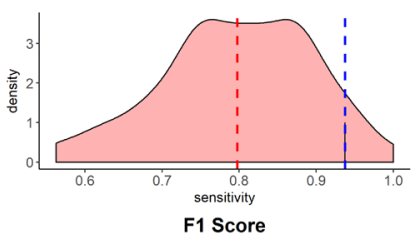

Data $=0.88$, Permuted Data Mean $=0.77$ $p$-value $=0.03$

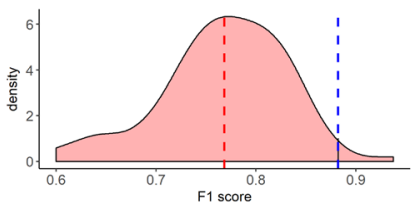

Positive Likelihood Ratio Data $=1.56$, Permuted Data Mean $=0.98$ $p$-value $=0.05$

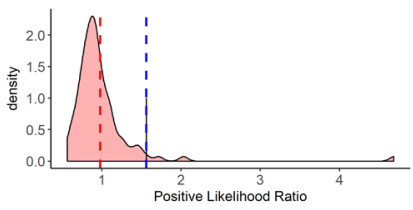

$\square$ Permuted data $\square$ Real data

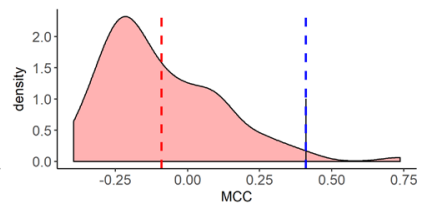

Negative Likelihood Ratio Data $=0.16$, Permuted Data Mean $=0.84$ $p$-value $=0.03$

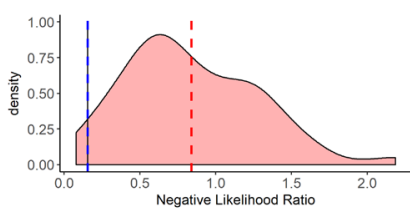

Figure 3

Classification results for Spain-Italy's original and permutated datasets using the random forest model. Density plots of statistical measures obtained by 100 iterations of the classification procedure on real (blue) and permutated (red) data sets. Permutated datasets included the same samples after random shuffling of their conditions (i.e., GDM/control). Permutations test means are indicated as well. Sensitivity: true positives out of all positives; Specificity: true negatives out of all negatives; Accuracy: true classifications out of all classifications; Matthews's correlation coefficient (MCC): a correlation coefficient between the observed and predicted binary classifications; AUC: area under the ROC curve; F1 Score: the harmonic mean of precision and sensitivity; Positive Likelihood Ratio: sensitivity / (1 - specificity); Negative Likelihood Ratio: (1 - sensitivity) / specificity.

prediction using several machine learning models that included all miRNAs (after applying a feature selection procedure), and tested their accuracy via a LOOCV procedure. We again evaluated the significance of the
Table 5 Maternal and pregnancy characteristics of the tissue samples cohort. Data are reported as medians (interquartile ranges).

\begin{tabular}{|c|c|c|c|}
\hline Characteristics & Control $(n=8)$ & GDM $(n=6)$ & $P$ value \\
\hline $\begin{array}{l}\text { Maternal age, } \\
\text { years }\end{array}$ & 36.5 (32.0-37.8) & $32.0(28.8-38.0)$ & 0.345 \\
\hline Gravity & $4.0(3.3-5.0)$ & $3.0(2.0-3.3)$ & 0.059 \\
\hline Parity & $1.5(0.3-3.8)$ & $1.5(0.8-2.0)$ & 0.662 \\
\hline $\begin{array}{l}\text { Body mass index, } \\
\mathrm{kg} / \mathrm{m}^{2}\end{array}$ & $26.7(22.5-29.3)$ & $27.0(21.0-36.5)$ & 0.950 \\
\hline
\end{tabular}

$P$ values were calculated using the Mann-Whitney $U$ test.

classification results using permutation tests. The latter achieved high performance and significant classification with all three models. More samples are required for a closer examination of the models' performances and for further comparison between them. Integrating clinical features with a known predictive value (e.g., adiponectin levels) to the classification model, can increase the classification accuracy even further.

The predictive value of microRNAs for GDM prediction was previously assessed in the second rather than in the first trimester $(49,50)$, and high variance of miRNA expression profiles was displayed between studies. Many potential explanations exist for this phenomenon. Undoubtedly there are true biological changes between

\section{A}

miR-223

miR-23a

\section{Placenta}
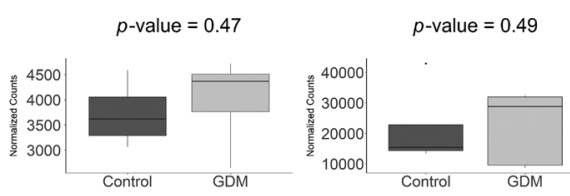

\section{Visceral \\ Adipose Tissue}

B
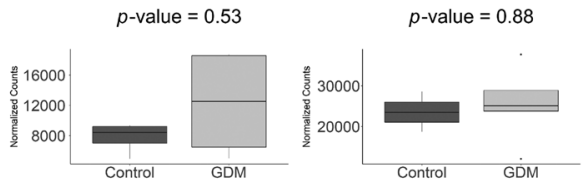

C

Adipose

Tissue
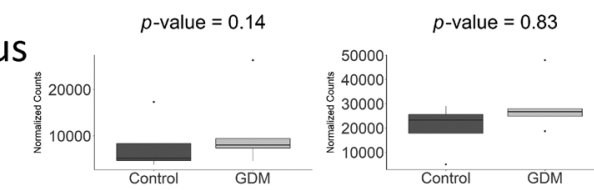

\section{Figure 4}

The expression of the differentially expressed miRNAs in the placenta and adipose tissues. Normalized counts of miR-223, and mir-23a in 3 tissue types obtained from GDM and control women: (A) placenta, (B) visceral adipose tissue, and (C) subcutaneous adipose tissue. Counts were obtained from NanoString nCounter miRNA profiling. 
women in different countries/ethnicities arising from genetic and environmental differences. It is well known that some populations have a higher risk to develop GDM than others. The dissimilarities in miRNA expression profiles may reflect true differences in GDM etiology. Additional possible explanation is technical noise that is unintentionally added to the miRNAs expression estimation as a result of small dissimilarities in the samples processing procedures, ranging from blood test technique to RNA extraction and miRNAs profiling. Each step in the processing chain adds variability to the final miRNA expression profile that in many cases obscures the true biological signal. This phenomenon termed 'batch effect' is well known in high-throughput experiments, and a plethora of statistical method was developed to adjust for this effect and to remove the unwanted noise from the data. Technical noise that is country-specific cannot be removed since it is impossible to distinguish it from the true biological differences between the populations. Hence, more samples from different clinics in each county are required to further characterize the differences between the countries. Maintaining a protocol for blood samples collection and handling, as well as for fasting condition can help to reduce irrelevant miRNA expression differences as well.

Our study has several limitations, which are derived mainly from the relatively limited number of samples in our dataset. The small-size dataset might lead to an overestimation of the model classification performance. To reduce this effect, we applied a LOOCV procedure and compared the performance to permutation tests. Nevertheless, we recognize that our results might still be overestimated. Acquiring more samples from different countries and ethnicities are required to validate the differentially expressed miRNAs and the performance of the classification models. Further studies are required to evaluate whether and to what extent the differentially expressed miRNAs in the first trimester are involved in GDM development. Since GDM symptoms appear in later stages of the pregnancy, it is important to explore miRNAs expression during the second and the third trimester as well and compare it to the expression profile of the first trimester. Such a comparison might shed light on the regulatory mechanisms and the involvement of miRNAs in GDM pathogenesis.

In summary, this study suggests that circulating miRNAs in maternal plasma can serve as predictive biomarkers for GDM. Those results should be validated on larger cohorts with samples from various origins. Our findings, which warrant further examination, lay the foundation for producing a novel early non-invasive screening test for GDM, which will serve as an effective intervention, and consequently reduce the risk of GDM and its sequelae for both the mother and offspring.

\section{Declaration of interest}

The authors declare that there is no conflict of interest that could be perceived as prejudicing the impartiality of the research reported.

\section{Funding}

LY is supported by the Eshkol Fellowship of the Israel Ministry of Science, Technology, and Space; and by the Edmond J. Safra Center for Bioinformatics at Tel Aviv University. The Shomron Laboratory is supported by the Israel Science Foundation (ISF; 1852/16); Israeli Ministry of Defense, Office of Assistant Minister of Defense for Chemical, Biological, Radiological and Nuclear (CBRN) Defense; Foundation Fighting Blindness; The Edmond J. Safra Center for Bioinformatics at Tel Aviv University; Zimin Institute for Engineering Solutions Advancing Better Lives; Eric and Wendy Schmidt Breakthrough Innovative Research Award; Tel Aviv University Richard Eimert Research Fund on Solid Tumors; Djerassi-Elias Institute of Oncology; and a generous donation from the Adelis Foundation.

\section{Author contribution statement}

LY, NS, and MH conceived and designed the study. MH, FM, AO, FC, EG, HK, and SMT acquired the data. $L Y, A P$, and $A G$ performed the experiments. LY and CR analyzed the data. LY and NS wrote the manuscript. NS and MH supervised the work and participated in the interpretation of the results. All authors critically reviewed the manuscript and approved its final version. LY, NS and $\mathrm{MH}$ are the guarantors of this work. N Shomron and M Hod contributed equally to this work.

\section{Acknowledgements}

The authors are indebted to the IDIBAPS Biobank, integrated in the Spanish National Biobank Network, for the sample and data procurement.

This work was performed in partial fulfillment of the requirements for a PhD degree of LY at the Sackler Faculty of Medicine, Tel Aviv University.

\section{References}

1 American Diabetes Association. Standards of medical care in diabetes-2018 abridged for primary care providers. Clinical Diabetes 201836 14-37. (https://doi.org/10.2337/cd17-0119)

2 Georgiou HM, Lappas M, Georgiou GM, Marita A, Bryant VJ, Hiscock R, Permezel M, Khalil Z \& Rice GE. Screening for biomarkers predictive of gestational diabetes mellitus. Acta Diabetologica $2008 \mathbf{4 5}$ 157-165. (https://doi.org/10.1007/s00592-008-0037-8)

3 Iliodromiti S, Sassarini J, Kelsey TW, Lindsay RS, Sattar N \& Nelson SM. Accuracy of circulating adiponectin for predicting gestational diabetes: a systematic review and meta-analysis. Diabetologia 201659 692-699. (https://doi.org/10.1007/s00125-0153855-6)

4 Catalano PM, Tyzbir ED, Wolfe RR, Calles J, Roman NM, Amini SB $\&$ Sims EA. Carbohydrate metabolism during pregnancy in control subjects and women with gestational diabetes. American Journal of Physiology 1993264 E60-E67. (https://doi.org/10.1152/ ajpendo.1993.264.1.E60)

5 Catalano PM, Huston L, Amini SB \& Kalhan SC. Longitudinal changes in glucose metabolism during pregnancy in obese women 
with normal glucose tolerance and gestational diabetes mellitus. American Journal of Obstetrics \& Gynecology 1999180 903-916. (https://doi.org/10.1016/s0002-9378(99)70662-9)

6 Kirwan JP, Mouzon SH-D, Lepercq J, Challier JC, Huston-Presley L, Friedman JE, Kalhan SC \& Catalano PM. TNF- $\alpha$ is a predictor of insulin resistance in human pregnancy. Diabetes 200251 2207-2213. (https://doi.org/10.2337/diabetes.51.7.2207)

7 Catalano PM, Tyzbir ED, Roman NM, Amini SB \& Sims EA. Longitudinal changes in insulin release and insulin resistance in nonobese pregnant women. American Journal of Obstetrics \& Gynecology 1991165 1667-1672. (https://doi.org/10.1016/00029378(91)90012-g)

8 Stanley K, Fraser R \& Bruce C. Physiological changes in insulin resistance in human pregnancy: longitudinal study with the hyperinsulinaemic euglycaemic clamp technique. British Journal of Obstetrics \& Gynaecology 1998105 756-759. (https://doi. org/10.1111/j.1471-0528.1998.tb10207.x)

9 Wolf M, Sandler L, Muñoz K, Hsu K, Ecker JL \& Thadhani R. First trimester insulin resistance and subsequent preeclampsia: a prospective study. Journal of Clinical Endocrinology \& Metabolism 2002 87 1563-1568. (https://doi.org/10.1210/jcem.87.4.8405)

10 Catalano PM. Trying to understand gestational diabetes. Diabetic Medicine 201431 273-281. (https://doi.org/10.1111/dme.12381)

11 Metzger BE, Buchanan TA, Coustan DR, Leiva A de, Dunger DB, Hadden DR, Hod M, Kitzmiller JL, Kjos SL, Oats JN, et al. Summary and recommendations of the fifth international workshop-conference on gestational diabetes mellitus. Diabetes Care 2007 30(Supplement 2) S251-S260. (https://doi.org/10.2337/ $\mathrm{dc07-s225)}$

12 Bowes SB, Hennessy TR, Umpleby AM, Benn JJ, Jackson NC, Boroujerdi MA, Sönksen PH \& Lowy C. Measurement of glucose metabolism and insulin secretion during normal pregnancy and pregnancy complicated by gestational diabetes. Diabetologia 199639 976-983. (https://doi.org/10.1007/bf00403918)

13 Hoffman L, Nolan C, Wilson JD, Oats JJ \& Simmons D. Gestational diabetes mellitus--management guidelines. The Australasian Diabetes in Pregnancy Society. Medical Journal of Australia 1998169 93-97. (https://doi.org/10.5694/j.1326-5377.1998.tb140192.x)

14 Rodrigo N \& Glastras SJ. The emerging role of biomarkers in the diagnosis of gestational diabetes mellitus. Journal of Clinical Medicine 20187 120. (https://doi.org/10.3390/jcm7060120)

15 Carr DB, Utzschneider KM, Hull RL, Tong J, Wallace TM, Kodama K, Shofer JB, Heckbert SR, Boyko EJ, Fujimoto WY, et al. Gestational diabetes mellitus increases the risk of cardiovascular disease in women with a family history of type 2 diabetes. Diabetes Care 2006 29 2078-2083. (https://doi.org/10.2337/dc05-2482)

16 Bellamy L, Casas JP, Hingorani AD \& Williams D. Type 2 diabetes mellitus after gestational diabetes: a systematic review and metaanalysis. Lancet 2009373 1773-1779. (https://doi.org/10.1016/ S0140-6736(09)60731-5)

17 Yang X, Hsu-Hage B, Zhang H, Zhang C, Zhang Y \& Zhang C. Women with impaired glucose tolerance during pregnancy have significantly poor pregnancy outcomes. Diabetes Care 200225 1619-1624. (https://doi.org/10.2337/diacare.25.9.1619)

18 Hillier TA, Pedula KL, Schmidt MM, Mullen JA, Charles MA \& Pettitt DJ. Childhood obesity and metabolic imprinting: the ongoing effects of maternal hyperglycemia. Diabetes Care 200730 2287-2292. (https://doi.org/10.2337/dc06-2361)

19 HAPO Study Cooperative Research Group, Metzger BE, Lowe LP, Dyer AR, Trimble ER, Chaovarindr U, Coustan DR, Hadden DR, McCance DR, Hod M, et al. Hyperglycemia and adverse pregnancy outcomes. New England Journal of Medicine 2008358 1991-2002. (https://doi.org/10.1056/NEJMoa0707943)

20 Crowther CA, Hiller JE, Moss JR, McPhee AJ, Jeffries WS, Robinson JS \& Australian Carbohydrate Intolerance Study in Pregnant Women (ACHOIS) Trial Group. Effect of treatment of gestational diabetes mellitus on pregnancy outcomes. New England Journal of Medicine 2005352 2477-2486. (https://doi.org/10.1056/NEJMoa042973)

21 Landon MB, Spong CY, Thom E, Carpenter MW, Ramin SM, Casey B, Wapner RJ, Varner MW, Rouse DJ, Thorp JM, et al. A multicenter, randomized trial of treatment for mild gestational diabetes. New England Journal of Medicine 2009361 1339-1348. (https://doi. org/10.1056/NEJMoa0902430)

22 Siegmund T, Rad NT, Ritterath C, Siebert G, Henrich W \& Buhling KJ. Longitudinal changes in the continuous glucose profile measured by the CGMS in healthy pregnant women and determination of cut-off values. European Journal of Obstetrics, Gynecology, and Reproductive Biology 2008139 46-52. (https://doi.org/10.1016/j. ejogrb.2007.12.006)

23 Committee on Practice Bulletins-Obstetrics. ACOG Practice Bulletin No. 190: Gestational diabetes mellitus. Obstetrics \& Gynecology 2018 131 e49-e64. (https://doi.org/10.1097/AOG.000000000002501)

24 Panel$^{*}$ IA of D and PSGC. International association of diabetes and pregnancy study groups recommendations on the diagnosis and classification of hyperglycemia in pregnancy. Diabetes Care 201033 676-682. (https://doi.org/10.2337/dc09-1848)

25 Agarwal MM, Punnose J \& Dhatt GS. Gestational diabetes: problems associated with the oral glucose tolerance test. Diabetes Research and Clinical Practice 200463 73-74. (https://doi.org/10.1016/j. diabres.2003.08.005)

26 Gillman MW, Oakey H, Baghurst PA, Volkmer RE, Robinson JS \& Crowther CA. Effect of treatment of gestational diabetes mellitus on obesity in the next generation. Diabetes Care 201033 964-968. (https://doi.org/10.2337/dc09-1810)

27 Plasencia W, Garcia R, Pereira S, Akolekar R \& Nicolaides KH. Criteria for screening and diagnosis of gestational diabetes mellitus in the first trimester of pregnancy. Fetal Diagnosis and Therapy 201130 108-115. (https://doi.org/10.1159/000324684)

28 Lain KY, Daftary AR, Ness RB \& Roberts JM. First trimester adipocytokine concentrations and risk of developing gestational diabetes later in pregnancy. Clinical Endocrinology 200869 407-411. (https://doi.org/10.1111/j.1365-2265.2008.03198.x)

29 International Expert Committee. International Expert Committee report on the role of the $\mathrm{A} 1 \mathrm{C}$ assay in the diagnosis of diabetes. Diabetes Care 200932 1327-1334. (https://doi.org/10.2337/dc099033)

30 Khalafallah A, Phuah E, Al-Barazan AM, Nikakis I, Radford A, Clarkson W, Trevett C, Brain T, Gebski V \& Corbould A. Glycosylated haemoglobin for screening and diagnosis of gestational diabetes mellitus. BMJ Open 20166 e011059. (https://doi.org/10.1136/ bmjopen-2016-011059)

31 Sweeting AN, Ross GP, Hyett J, Molyneaux L, Tan K, Constantino M, Harding AJ \& Wong J. Baseline HbA1c to identify high-risk gestational diabetes: utility in early vs standard gestational diabetes. Journal of Clinical Endocrinology \& Metabolism 2017102 150-156. (https://doi.org/10.1210/jc.2016-2951)

32 O'Connor C, O'Shea PM, Owens LA, Carmody L, Avalos G, Nestor L, Lydon K \& Dunne F. Trimester-specific reference intervals for haemoglobin A1c (HbA1c) in pregnancy. Clinical Chemistry and Laboratory Medicine 201150 905-909. (https://doi.org/10.1515/ CCLM.2011.397)

33 Corcoran SM, Achamallah N, Loughlin JO, Stafford P, Dicker P, Malone FD \& Breathnach F. First trimester serum biomarkers to predict gestational diabetes in a high-risk cohort: striving for clinically useful thresholds. European Journal of Obstetrics, Gynecology, and Reproductive Biology 2018222 7-12. (https://doi.org/10.1016/j. ejogrb.2017.12.051)

34 Syngelaki A, Visser GHA, Krithinakis K, Wright A \& Nicolaides KH. First trimester screening for gestational diabetes mellitus by maternal factors and markers of inflammation. Metabolism: Clinical \& Experimental 201665 131-137. (https://doi.org/10.1016/j. metabol.2015.10.029) 
35 Castillo-Castrejon M \& Powell TL. Placental nutrient transport in gestational diabetic pregnancies. Frontiers in Endocrinology 20178 306. (https://doi.org/10.3389/fendo.2017.00306)

36 He L \& Hannon GJ. MicroRNAs: small RNAs with a big role in gene regulation. Nature Reviews. Genetics 20045 522-531. (https://doi. org/10.1038/nrg1379)

37 Shen Q \& Temple S. Fine control: microRNA regulation of adult neurogenesis. Nature Neuroscience 200912 369-370. (https://doi. org/10.1038/nn0409-369)

38 Esquela-Kerscher A \& Slack FJ. Oncomirs - microRNAs with a role in cancer. Nature Reviews. Cancer 20066 259-269. (https://doi. org/10.1038/nrc1840)

39 Hammond SM. MicroRNAs as tumor suppressors. Nature Genetics 200739 582-583. (https://doi.org/10.1038/ng0507-582)

40 Croce CM. Causes and consequences of microRNA dysregulation in cancer. Nature Reviews. Genetics 200910 704-714. (https://doi. org/10.1038/nrg2634)

41 Nicoloso MS, Spizzo R, Shimizu M, Rossi S \& Calin GA. MicroRNAs-the micro steering wheel of tumour metastases. Nature Reviews. Cancer 20099 293-302. (https://doi.org/10.1038/nrc2619)

42 Peng Y \& Croce CM. The role of microRNAs in human cancer. Signal Transduction and Targeted Therapy 20161 15004. (https://doi. org/10.1038/sigtrans.2015.4)

43 Sebastiani G, Nigi L, Grieco GE, Mancarella F, Ventriglia G \& Dotta F Circulating microRNAs and diabetes mellitus: a novel tool for disease prediction, diagnosis, and staging? Journal of Endocrinological Investigation 201740 591-610. (https://doi.org/10.1007/s40618-0170611-4)

44 Zampetaki A, Kiechl S, Drozdov I, Willeit P, Mayr U, Prokopi M, Mayr A, Weger S, Oberhollenzer F, Bonora E, et al. Plasma microRNA profiling reveals loss of endothelial MiR-126 and other microRNAs in type 2 diabetes novelty and significance. Circulation Research 2010 107 810-817. (https://doi.org/10.1161/CIRCRESAHA.110.226357)

45 Jiménez-Lucena R, Rangel-Zúñiga OA, Alcalá-Díaz JF, LópezMoreno J, Roncero-Ramos I, Molina-Abril H, Yubero-Serrano EM, Caballero-Villarraso J, Delgado-Lista J, Castaño JP, et al. Circulating miRNAs as predictive biomarkers of type 2 diabetes mellitus development in coronary heart disease patients from the CORDIOPREV study. Molecular Therapy. Nucleic Acids 201812 146-157. (https://doi.org/10.1016/j.omtn.2018.05.002)

46 Guay C \& Regazzi R. New emerging tasks for microRNAs in the control of $\beta$-cell activities. Biochimica et Biophysica Acta 20161861 2121-2129. (https://doi.org/10.1016/j.bbalip.2016.05.003)

47 Ventriglia G, Nigi L, Sebastiani G \& Dotta F. MicroRNAs: novel players in the dialogue between pancreatic islets and immune system in autoimmune diabetes. BioMed Research International 20152015 749734. (https://doi.org/10.1155/2015/749734)

48 Sebastiani G, Po A, Miele E, Ventriglia G, Ceccarelli E, Bugliani M, Marselli L, Marchetti P, Gulino A, Ferretti E, et al. MicroRNA-124a is hyperexpressed in type 2 diabetic human pancreatic islets and negatively regulates insulin secretion. Acta Diabetologica 201552 523-530. (https://doi.org/10.1007/s00592-014-0675-y)

49 Zhao C, Dong J, Jiang T, Shi Z, Yu B, Zhu Y, Chen D, Xu J, Huo R, Dai J, et al. Early second-trimester serum MiRNA profiling predicts gestational diabetes mellitus. PLOS ONE 20116 e23925. (https://doi. org/10.1371/journal.pone.0023925)

50 Zhu Y, Tian F, Li H, Zhou Y, Lu J \& Ge Q. Profiling maternal plasma microRNA expression in early pregnancy to predict gestational diabetes mellitus. International Journal of Gynaecology and Obstetrics 2015130 49-53. (https://doi.org/10.1016/j.ijgo.2015.01.010)

51 Zhao C, Zhang T, Shi Z, Ding H \& Ling X. MicroRNA-518d regulates PPAR $\alpha$ protein expression in the placentas of females with gestational diabetes mellitus. Molecular Medicine Reports 20149 2085-2090. (https://doi.org/10.3892/mmr.2014.2058)

52 Li J, Song L, Zhou L, Wu J, Sheng C, Chen H, Liu Y, Gao S \& Huang W. A microRNA signature in gestational diabetes mellitus associated with risk of macrosomia. Cellular Physiology and Biochemistry 201537 243-252. (https://doi.org/10.1159/000430349)

53 Shi Z, Zhao C, Guo X, Ding H, Cui Y, Shen R \& Liu J. Differential expression of microRNAs in omental adipose tissue from gestational diabetes mellitus subjects reveals miR-222 as a regulator of ER $\alpha$ expression in estrogen-induced insulin resistance. Endocrinology 2014 155 1982-1990. (https://doi.org/10.1210/en.2013-2046)

54 Floris I, Descamps B, Vardeu A, Mitić T, Posadino AM, Shantikumar S, Sala-Newby G, Capobianco G, Mangialardi G, Howard L, et al. Gestational diabetes mellitus impairs fetal endothelial cell functions through a mechanism involving microRNA-101 and histone methyltransferase enhancer of zester homolog-2. Arteriosclerosis, Thrombosis, \& Vascular Biology 201535 664-674. (https://doi. org/10.1161/ATVBAHA.114.304730)

55 Tryggestad JB, Vishwanath A, Jiang S, Mallappa A, Teague AM, Takahashi Y, Thompson DM \& Chernausek SD. Influence of gestational diabetes mellitus on human umbilical vein endothelial cell miRNA. Clinical Science 2016130 1955-1967. (https://doi. org/10.1042/CS20160305)

56 Geiss GK, Bumgarner RE, Birditt B, Dahl T, Dowidar N, Dunaway DL, Fell HP, Ferree S, George RD, Grogan T, et al. Direct multiplexed measurement of gene expression with color-coded probe pairs. Nature Biotechnology 200826 317-325. (https://doi.org/10.1038/ nbt1385)

57 Love MI, Huber W \& Anders S. Moderated estimation of fold change and dispersion for RNA-seq data with DESeq2. Genome Biology 2014 15 550. (https://doi.org/10.1186/s13059-014-0550-8)

58 Breiman L. Random forests. Machine Learning 200145 5-32. (https:// doi.org/10.1023/A:1010933404324)

59 Freund Y \& Schapire RE. A decision-theoretic generalization of on-line learning and an application to boosting. Journal of Computer and System Sciences 199755 119-139. (https://doi.org/10.1006/ jcss.1997.1504)

60 Davison AC \& Hinkley D. Bootstrap methods and their application. Journal of the American Statistical Association 199794.

61 Ritchie ME, Phipson B, Wu D, Hu Y, Law CW, Shi W \& Smyth GK. limma powers differential expression analyses for RNA-sequencing and microarray studies. Nucleic Acids Research 201543 e47. (https:// doi.org/10.1093/nar/gkv007)

62 Pedregosa F, Varoquaux G, Gramfort A, Michel V, Thirion B, Grisel O, Blondel M, Prettenhofer P, Weiss R, Dubourg V, et al. Scikit-learn: machine Learning in Python. Journal of Machine Learning Research 201112 2825-2830.

63 Livak KJ \& Schmittgen TD. Analysis of relative gene expression data using real-time quantitative PCR and the $2-\Delta \Delta$ CT method. Methods 200125 402-408. (https://doi.org/10.1006/meth.2001.1262)

64 Fulci V, Scappucci G, Sebastiani GD, Giannitti C, Franceschini D, Meloni F, Colombo T, Citarella F, Barnaba V, Minisola G, et al. miR-223 is overexpressed in T-lymphocytes of patients affected by rheumatoid arthritis. Plos One 201071 206-211. (https://doi. org/10.1016/j.humimm.2009.11.008)

65 Fasseu M, Tréton X, Guichard C, Pedruzzi E, Cazals-Hatem D, Richard C, Aparicio T, Daniel F, Soulé JC, Moreau R, et al. Identification of restricted subsets of mature microRNA abnormally expressed in inactive colonic mucosa of patients with inflammatory bowel disease. PLOS ONE 2010 5. (https://doi.org/10.1371/journal. pone.0013160)

66 Li Y, Chan EY, Li J, Ni C, Peng X, Rosenzweig E, Tumpey TM \& Katze MG. MicroRNA expression and virulence in pandemic influenza virus-infected mice. Journal of Virology $2010843023-3032$. (https://doi.org/10.1128/JVI.02203-09)

$67 \mathrm{Xu} \mathrm{J}, \mathrm{Wu}$ C, Che X, Wang L, Yu D, Zhang T, Huang L, Li H, Tan W, Wang C, et al. Circulating microRNAs, miR-21, miR-122, and miR223 , in patients with hepatocellular carcinoma or chronic hepatitis. Molecular Carcinogenesis 201150 136-142. (https://doi.org/10.1002/ mc.20712) 
68 Stamatopoulos B, Meuleman N, Haibe-Kains B, Saussoy P, Van Den Neste E, Michaux L, Heimann P, Martiat P, Bron D \& Lagneaux L. microRNA-29c and microRNA-223 down-regulation has in vivo significance in chronic lymphocytic leukemia and improves disease risk stratification. Blood 2009113 5237-5245. (https://doi. org/10.1182/blood-2008-11-189407)

69 Imig J, Motsch N, Zhu JY, Barth S, Okoniewski M, Reineke T, Tinguely M, Faggioni A, Trivedi P, Meister G, et al. MicroRNA profiling in Epstein-Barr virus-associated B-cell lymphoma. Nucleic Acids Research 201139 1880-1893. (https://doi.org/10.1093/nar/ gkq1043)

$70 \mathrm{Lu} \mathrm{H}$, Buchan RJ \& Cook SA. MicroRNA-223 regulates Glut4 expression and cardiomyocyte glucose metabolism. Cardiovascular Research 201086 410-420. (https://doi.org/10.1093/cvr/cvq010)

71 Liang YZ, Li JJ, Xiao HB, He Y, Zhang L \& Yan YX. Identification of stress-related microRNA biomarkers in type 2 diabetes mellitus: a systematic review and meta-analysis. Journal of Diabetes 2018. (https://doi.org/10.1111/1753-0407.12643)

72 Wander PL, Boyko EJ, Hevner K, Parikh VJ, Tadesse MG, Sorensen TK, Williams MA \& Enquobahrie DA. Circulating earlyand mid-pregnancy microRNAs and risk of gestational diabetes. Diabetes Research and Clinical Practice 2017132 1-9. (https://doi. org/10.1016/j.diabres.2017.07.024)

73 Hromadnikova I, Kotlabova K, Hympanova L \& Krofta L. Gestational hypertension, preeclampsia and intrauterine growth restriction induce dysregulation of cardiovascular and cerebrovascular disease associated microRNAs in maternal whole peripheral blood. Thrombosis Research 2016137 126-140. (https://doi.org/10.1016/j.thromres.2015.11.032)

74 Yang Z, Chen H, Si H, Li X, Ding X, Sheng Q, Chen P \& Zhang H. Serum miR-23a, a potential biomarker for diagnosis of pre-diabetes and type 2 diabetes. Acta Diabetologica 201451 823-831. (https://doi. org/10.1007/s00592-014-0617-8)

Received 18 March 2019

Revised version received 12 September 2019

Accepted 17 September 2019 